Barreda Usó, Gemma.

Conservadora-Restauradora, Doctora en Bellas Artes.

Zalbidea Muñoz, Mạ Antonia.

Universitat Politècnica de València. Profesora Titular del Departamento

de Conservación y Restauración de Bienes Culturales.

Osca Pons, Julia.

Universitat Politècnica de València. Profesora Titular del Departamento

de Conservación y Restauración de Bienes Culturales.

\title{
Comparativa entre distintos consolidantes inorgánicos nanoparticulados a base de hidróxido cálcico.
}

\section{Comparison between different inorganic nanoparticulate consolidators based on calcium hydroxide.}

TIPO DE TRABAJO:

Comunicación.

PALABRAS CLAVE:

Consolidación, consolidantes inorgánicos, nanopartículas, hidróxido cálcico, material pétreo.

KEY WORDS:

Consolidation, inorganic consolidantes, nanoparticles, calcium hydroxide, stone material.

RESUMEN.

El presente trabajo de investigación muestra el estudio comparativo realizado entre diversos materiales consolidantes nanoparticulados de origen inorgánico a base de hidróxido cálcico, aplicados sobre soportes pétreos con matriz carbónica.

Para realizar esta comparativa se han seleccionado diversos materiales nanoestructurados que han sido diseñados expresamente para la conservación del patrimonio, concretamente para la consolidación de soportes pétreos, morteros, pintura mural, etc. Debido a la novedad de estos productos, es necesario su estudio individualizado previamente a ser usados en ciertos campos de la restauración como sucede con la consolidación de pintura rupestre realizada sobre roca calcárea. Por ello se seleccionaron los posibles productos afines a esta necesidad.

Los consolidantes elegidos para realizar el proceso de testado son materiales nanoparticulados basados en soluciones coloidales de hidróxido cálcico $\mathrm{Ca}(\mathrm{OH})_{2}$ dispersos en diferentes tipos de alcoholes y en distintas concentraciones. Entre ellos encontramos, para tratamientos de consolidación superficial, descohesión o pulverulencia, el Nanorestore ${ }^{\circledR}$ y los productos de la gama CaLoSiL ${ }^{\circledR}$; en distintas variantes como: CaLoSiL E5 ${ }^{\circledR}$, CaLoSiL E25 grey $^{\circledR}, \mathrm{CaLoSiL} \mathrm{IP5}^{\circledR}, \mathrm{CaLoSiL} \mathrm{NP5}^{\circledR}$; y para tratamientos de adhesión entre estratos el CaLoSiL paste like ${ }^{\circledR}$ y el CaLoSiL Micro ${ }^{\circledR}$.

Tan sólo con los resultados obtenidos, se podrá determinar si estos nanoparticulados, son adecuados para su uso en obra real con unas características concretas. 


\section{ABSTRACT.}

The present research work shows the comparative study carried out between different nanoparticulate consolidating materials of inorganic origin based on calcium hydroxide, on stony supports with carbonic matrix.

In order to carry out this comparison, various nanostructured materials have been selected which have been specifically designed for the preservation of the heritage, specifically for the consolidation of stone supports, mortars, mural painting, etc. Due to the novelty of these products, it is necessary to study them individually before being used in certain fields of the restoration as it happens with the consolidation of rock painting done on calcareous rock. For this reason, the possible products related to this need were selected.

The consolidants chosen to carry out the test process are nanoparticulate materials based on colloidal solutions of calcium hydroxide $\mathrm{Ca}(\mathrm{OH})_{2}$ dispersed in different types of alcohols and in different concentrations. Among them, we find, for treatments of surface consolidation, the Nanorestore ${ }^{\circledR}$ and the products of the range CaLoSiL ${ }^{\oplus}$; Within it have been tested different variants such as: CaLoSiL E5 ${ }^{\circledR}$, CaLoSiL E25 grey $^{\circledR}$, CaLoSiL IP5 ${ }^{\circledR}$, CaLoSiL NP5 ${ }^{\circledR}$; and for adhesion treatments between strata, CaLoSiL paste like ${ }^{\circledR}$ and CaLoSiL Micro ${ }^{\circledR}$ have been chosen.

Only with the results obtained, it will be possible to determine if these nanoparticles are suitable for use in real work with specific characteristics.

\section{CONTENIDO.}

\section{INTRODUCCIÓN.}

El origen de esta investigación es determinar y estudiar las características concretas de distintos consolidantes inorgánicos nanopartículados a base de hidróxido cálcico, productos elaborados en los últimos años con el desarrollo de la nanociencia y la nanotecnología destinados a ser utilizados en el campo de la conservación y restauración del patrimonio. Estos materiales nanoestructurados confieren un comportamiento totalmente diverso a los materiales tradicionales, proporcionandoles características funcionales y estructurales innovadoras. Los consolidantes elegidos para realizar el proceso de análisis son materiales nanoparticulados a base hidróxido cálcico $\mathrm{Ca}(\mathrm{OH})_{2}$ disperso en soluciones coloidales con diferentes tipos de alcoholes y en distintas concentraciones. Entre ellos se han seleccionado para tratamientos de consolidación superficial, descohesión o pulverulencia, el Nanorestore ${ }^{\circledR}$ y los productos de la gama CaLoSiL ${ }^{\circledR}$ (CaLoSiL E5 ${ }^{\oplus}$, CaLoSiL E25 grey ${ }^{\circledast}$, CaLoSiL IP5 ${ }^{\oplus}$, CaLoSiL NP5 ${ }^{\circledR}$ ) y para realizar tratamientos de adhesión entre estratos se han escogido el CaLoSiL paste like ${ }^{\circledR}$ y el CaLoSiL Micro ${ }^{\circledast}$.

La ventaja de estos productos radica en su posible aplicación como consolidante de superficies pétreas con manifestaciones de arte rupestre. Para poder hacer uso de estos productos en obra real previamente requieren ser testados y analizados individualmente para conocer sus posibilidades y su repercusión con el soporte. Demás se recuerda que se requiere de una exhaustiva investigación científica previa a cualquier intervención sobre pintura rupestre para garantizar la correcta conservación de la obra.

\section{CONSOLIDANTES NANOPARTICULADOS.}

Actualmente con el desarrollo de la nanociencia y la nanotecnología se ha conseguido la creación de diferentes materiales con características funcionales y estructurales muy novedosas. Estos consolidantes están constituidos por partículas nano-estructuradas de cal apagada dispersas en alcohol. El alcohol, garantiza una óptima penetración en la matriz porosa, quedando las nanopartículas insertadas en los intersticios y en la red porosa a consolidar. Una vez transformados en carbonato de calcio por acción del anhídrido carbónico atmosférico, originan una red de micro-cristales de calcita que confieren elevadas propiedades mecánicas sin introducir materiales extraños a la naturaleza química de la roca. Las partículas a escala nanométrica confieren a estos productos un comportamiento diverso a los materiales tradicionales que por lo general han sido polímeros sintéticos constituidos por macromoléculas.

Estudios previos han sido concluyentes para afirmar científicamente que los mejores resultados obtenidos para la consolidación de soportes pétreos con arte rupestre se han obtenido con la aplicación y uso de materiales inorgánicos (Barreda, Gemma: 2016). Los productos inorgánicos utilizados en procesos de consolidación y fijación de soporte pétreo, funcionan precipitando un compuesto inorgánico en el interior de la estructura porosa del material tratado. La ventaja más favorable para el uso de productos inorgánicos es la compatibilidad con la naturaleza del soporte, que no produce impermeabilización pero sí disminución del tamaño del poro del material. Los productos inorgánicos tradicionales, presentan ciertas desventajas: el elevado tiempo para efectuar la consolidación con respecto a las resinas sintéticas; el aporte de gran cantidad de agua en periodos prolongados que puede provocar solubilización de 
sales y eflorescencias; además de la facilidad para formar velos blanquecinos difíciles de eliminar. Estos inconvenientes se minimizan con el uso de productos como el Nanorestore ${ }^{\circledR}$ (DEI, Luigi: 2006) o CaLoSiL ${ }^{\circledR}$ (ZIEGENBALB: 2008) cuyas propiedades técnicas restringen la aparición de velos blanqucinos, además el aporte de humedad es inferior debido ya que al se dispersiones alcoholicas favorecen la penetración.

La aplicación de nanopartículas ${ }^{1}$ posibilita el uso de tratamientos de protección, mediante la mejora del grado de recubrimiento, penetración, métodos de aplicación y la eficacia de los tratamientos de hidrofugación, permitiendo desarrollar nuevos productos hidrorrepelentes con propiedades auto-limpiantes que disminuyen el mantenimiento de los materiales y aumentan su durabilidad ${ }^{2}$.

El hidróxido de cal es uno de los materiales tradicionales de origen inorgánico más utilizado y conocido, utilizado para solucionar la descohesión del material pétreo a lo largo de la historia. Éste, al entrar en contacto con el $\mathrm{CO}_{2}$ atmosférico carbonata, reaccionando y transformándose en carbonato cálcico, material compatible con la composición pétrea de matriz cálcica.

Para el estudio se han utilizado dispersiones de $5 \mathrm{~g}$ de hidróxido cálcico por litro de alcohol, que favorecen la penetración y evitan la readhesión entre las mismas nanopartículas de $\mathrm{Ca}(\mathrm{OH})_{2}$. Ya que en las dispersiones muy concentradas se crean conglomeraciones que dificultan la penetración a través de la estructura porosa quedando retenidas en superficie, generando un efecto óptico-estético no deseable en forma de veladuras (velos blanquecinos) ${ }^{3}$.

Todos los productos testados son dispersiones en diferentes alcoholes. Los alcoholes garantizan una óptima penetración por succión capilar en los materiales porosos, gracias a su baja tensión superficial. Hecho que favorece el transporte de las nanopartículas a través de la matriz porosa, quedando insertadas en los intersticios y en las zonas situadas inmediatamente por debajo de la superficie.

Los consolidantes denominados CaLoSiL ${ }^{\circledR}$ han sido sintetizados como consolidantes para piedra y revoques. El disolvente se evapora sin dejar residuos y no se produce la formación de sub-productos, ni elementos químicos que puedan ocasionar alteraciones. La velocidad de evaporación del disolvente es un factor a tener en cuenta, ya que interviene en el transporte de las partículas de hidróxido cálcico y en la penetración. Siendo el alcohol N-propanol de evaporación más lenta que el Isopropanol seguido del Etanol.

Durante el proceso de carbonatación se produce un incremento de masa y volumen. Además intervenen otros factores como:

- $\quad$ La composición mineralógica del sustrato.

- $\quad$ El contenido en humedad de la piedra.

- $\quad$ El nivel de deterioro.

- La absorbencia.

- $\quad$ La porosidad del soporte.

- $\quad$ La absorción de agua por higroscopicidad.

Generalmente tras el proceso de carbonatación se forma Calcita $\left(\mathrm{Ca} \mathrm{CO}_{3}\right)$, caracterizada por su estabilidad, pero según las condiciones del soporte se puede formar Vaterita o Aragonita (GÓMEZ, Luz Stella: 2012. pp. 63), minerales poliformos que tienen la misma fórmula química pero distinta estructura cristalina, caracterizados por su inestabilidad y solubilidad ${ }^{4}$.

\footnotetext{
${ }^{1}$ El estudio del efecto de los productos consolidantes basados en nanopartículas (DANIELE, Valeria. et al.: 2008, 2010; LÓPEZ-ARCE, Paula. et al.: 2010; GÓMEZ, Luz Stella. et al: 2010-11-12) obtenidos por diferentes métodos de síntesis aplicados con el fin de minimizar el deterioro de los geomateriales permite evaluar su comportamiento a nanoescala y su compatibilidad con el material original, su evolución morfológica, la variación cristalina y analizar su reacción con diversas superficies a las que han sido expuestas. Otro estudio hace posible la valoración del comportamiento de estos consolidantes en diferentes condiciones ambientales, donde la humedad relativa, la temperatura y el tiempo de exposición al producto controlarán su eficacia y durabilidad (Gómez, Luz Stella et al: 2010).

${ }^{2}$ GÓMEZ, Luz Stella, et al. La aportación de la nanociencia a la conservación de bienes del patrimonio cultural. Patrimonio cultural de España, no. 4, 2010. pp. 50-51.

${ }^{3} \mathrm{DEI}$, Luigi, et al. Pre-consolidation of pictorial layers in frescoes: The high performance of CSGI's method based on nanolime evaluated by opd team in agnolo gaddi's Leggenda della Vera Croce Paintings, Santa Croce. En Scienza e Beni Culturali, XXIII. Florence, 2007. pp. 217-223.

${ }^{4}$ Jornada CaLoSiL®. Universitat Politècnica de València, 24 de mayo de 2013. Organizada por J. Osca.
} 
Barreda Usó, Gemma; Zalbidea Muñoz, Mạ Antonia; Osca Pons, Julia

Comparativa entre distintos consolidantes inorgánicos nanoparticulados a base de hidróxido cálcico

III CONGRESO INTERNACIONAL DE INVESTIGACIÓN EN ARTES VISUALES :: ANIAV 2017 :: GLOCAL [codificar, mediar, transformar, vivir] http://dx.doi.org/10.4995/ANIAV.2017.5702

El CaLoSiL $E^{\circledR}$ (Ilustración 1) es una dispersión de nano hidróxido de calcio en etanol. Diseñada para ser utilizada en tratamientos de consolidación próximas a la superficie. Se comercializa en distintas concentraciones: E5 (5 g/l), E25 (25 g/l) y E50 (50 g/l).

El CaLoSiL E25 grey ${ }^{\circledR}$ (Ilustración 2) es una dispersión de nano-cal $\left(\mathrm{Ca}(\mathrm{OH})_{2}\right)$ en Etanol. Diseñado para tratamientos de superficies delicadas que presenten descohesión. El tamaño de las partículas oscila entre los 50 y los $250 \mathrm{~nm}$, y se presenta en concentraciones de $25 \mathrm{~g} / \mathrm{l}$. Según el fabricante, debido precisamente a su consistencia más diluida, penetra más y más rápido, incluso en estructuras densas y poco porosas.

El CaLoSiL IP5 ${ }^{\circledR}$ (Ilustración 3) es una dispersión de hidróxido de calcio en Isopropanol, se comercializa en distintas concentraciones. Ha sido creado para se aplicado en tratamientos superficiales de pinturas al fresco, superficies pulverulentas, morteros, escayolas y piedras menos porosas, debido a la baja concentración de sólido $\left(\mathrm{Ca}(\mathrm{OH})_{2}\right)$ favorece la consolidación en estos casos concreto.

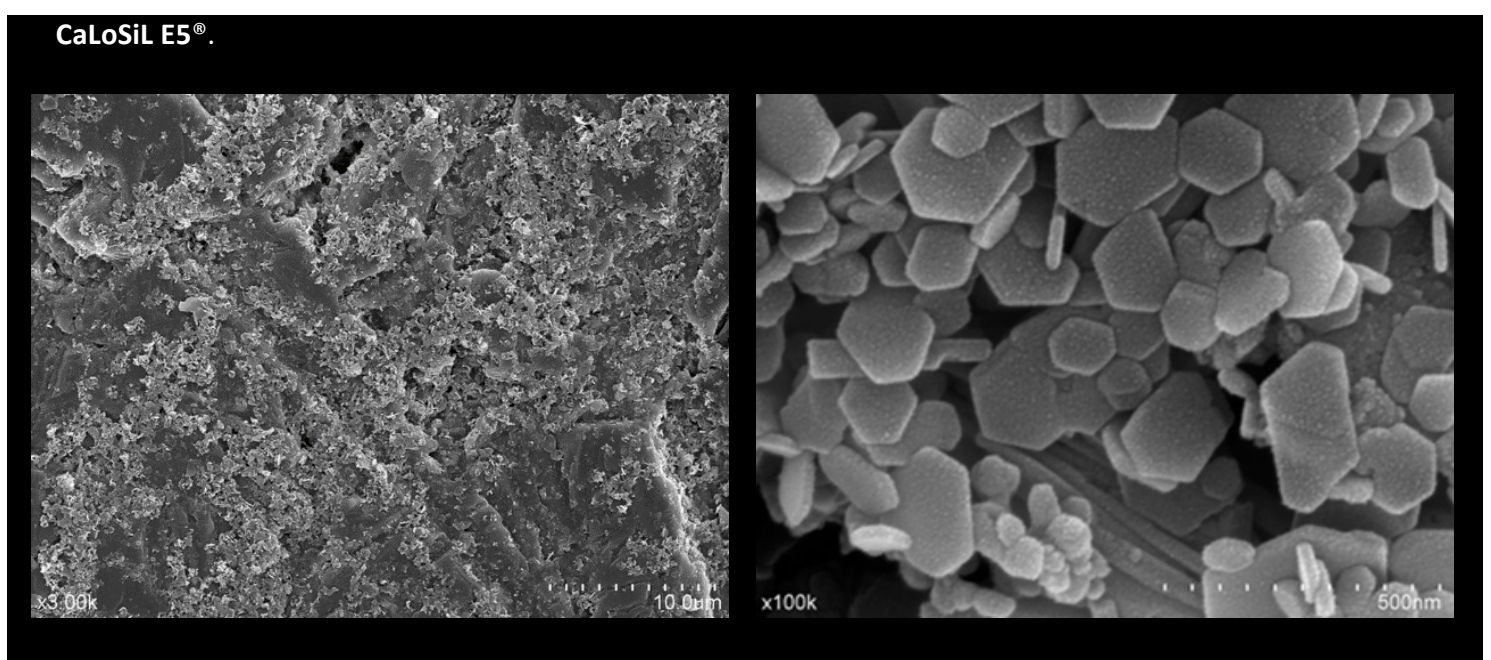

Ilustración 1. CaLoSiL E5 ${ }^{\circledR}$. SEM-(Hitachi S4800).

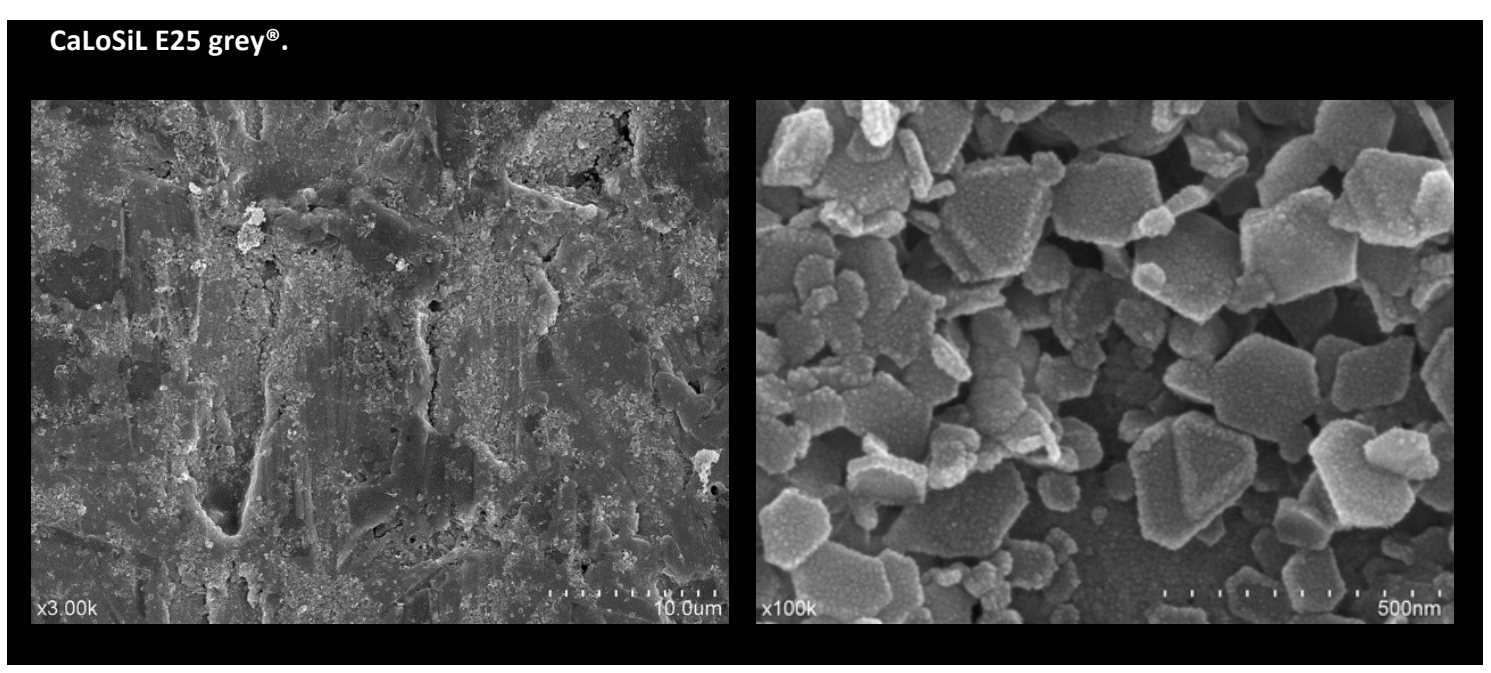

Ilustración 2. CaLoSiL E25 grey ${ }^{\circledR}$. SEM-(Hitachi S4800). 


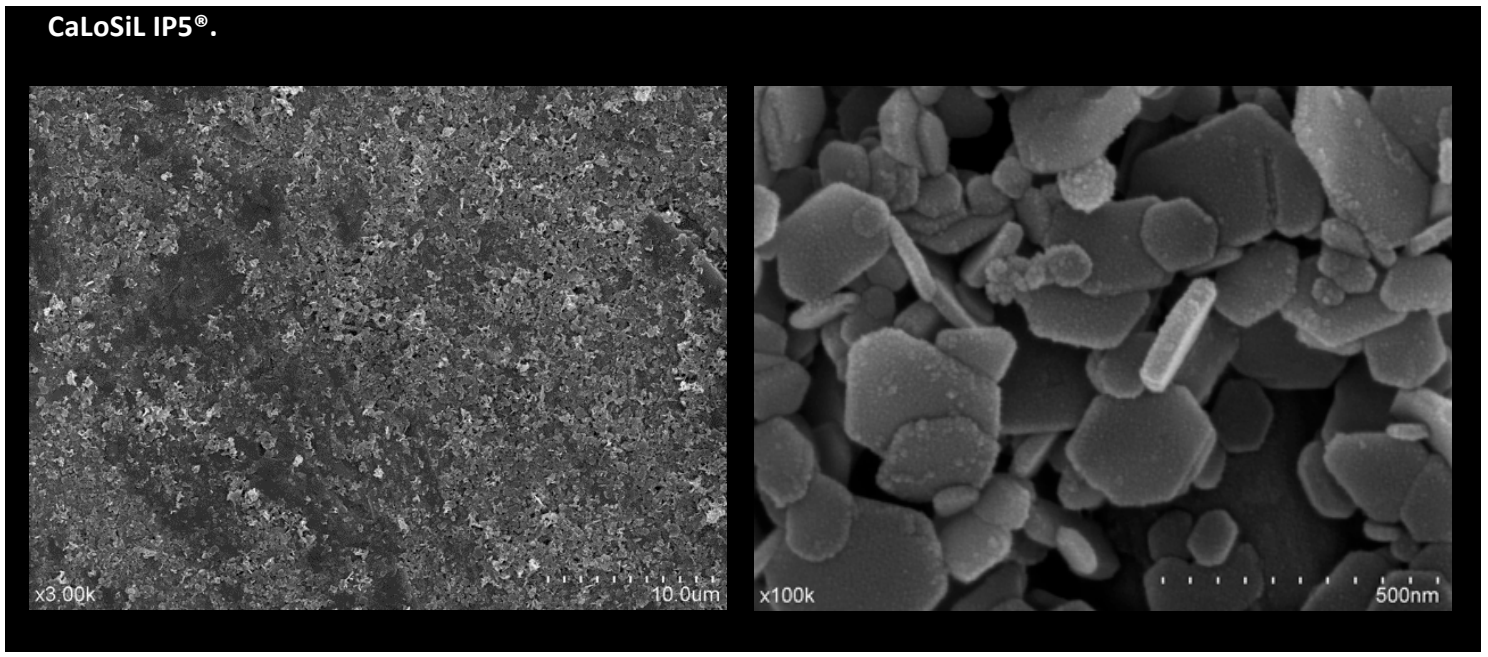

\section{Ilustración 3. CaLoSiL IP5 ${ }^{\circledR}$. SEM-(Hitachi S4800).}

El CaLoSiL NP5 ${ }^{\circledR}$ (Ilustración 4) es una dispersión de hidróxido de cal en 1-Propanol, alcohol que confiere la propiedad de evaporar lentamente, se utiliza para consolidar zonas profundas, ya que su lenta evaporación, facilita la penetración del material. En los casos anteriores la evaporación más rápida del disolvente hace que disminuya la penetración.

El Nanorestore ${ }^{\circledR}$ (Ilustración 5) es una dispersión de hidróxido de cal en alcohol Isopropílico. Estudiado en investigaciones anteriores ${ }^{5}$ para tratamientos de consolidación de material de origen calcáreo-dolomítico, con manifestaciones de arte rupestre. El resultado ofreciendo por este producto para realizar tratamientos de consolidación en superficie ha sido satisfactorio. Sus propiedades le confieren una excelente compatibilidad con soportes pétreos de naturaleza carbonática; por su permeabilidad al vapor de agua, la ausencia de variaciones cromáticas o efectos de brillo, además de mantener la hidrofilia del soporte a tratar, sin generar películas superficiales hidrófugas, ni velos blanquecinos.

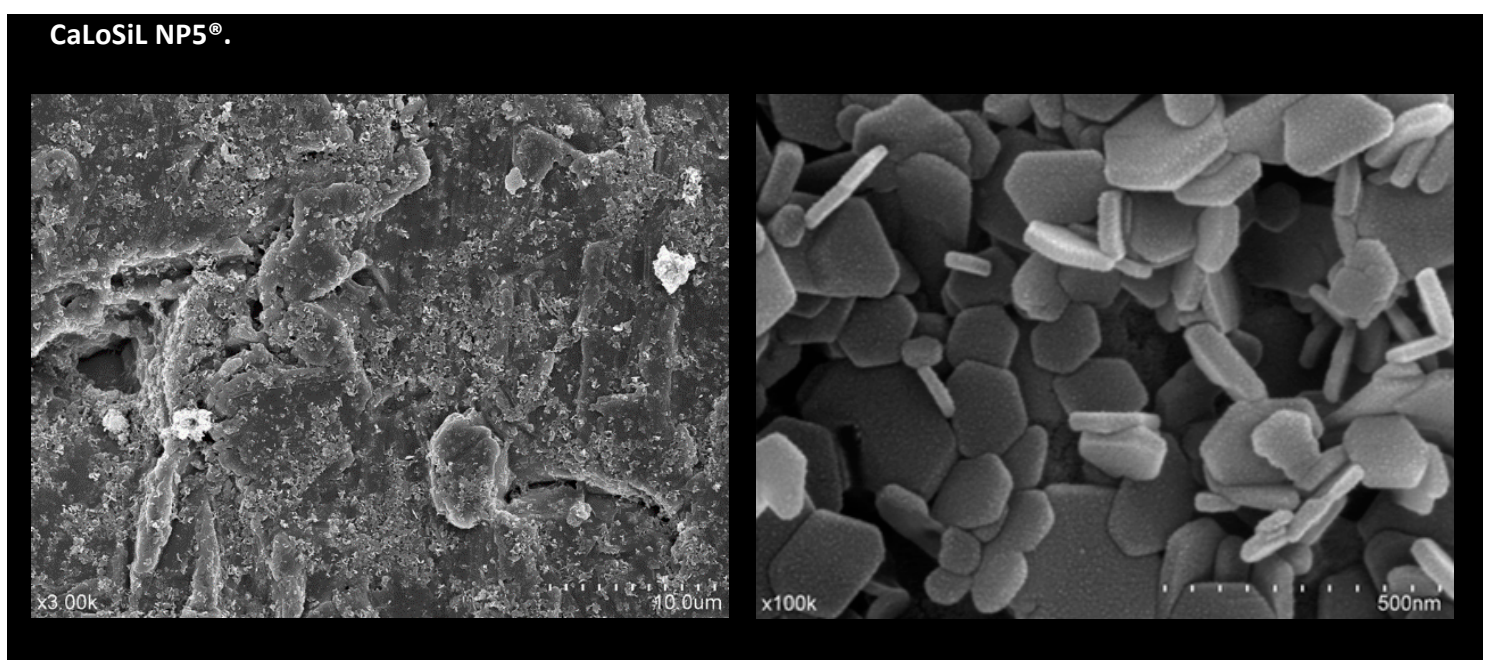

Ilustración 4. CaLoSiL NP5 ${ }^{\circledR}$. SEM-(Hitachi S4800).

\footnotetext{
${ }^{5}$ Barreda, Gemma. Investigación de tratamientos de consolidación del soporte rocoso en el Abric de Pinos (Benissa-Alicante), TFM, Universitat Politècnica de València. 2012.
} 


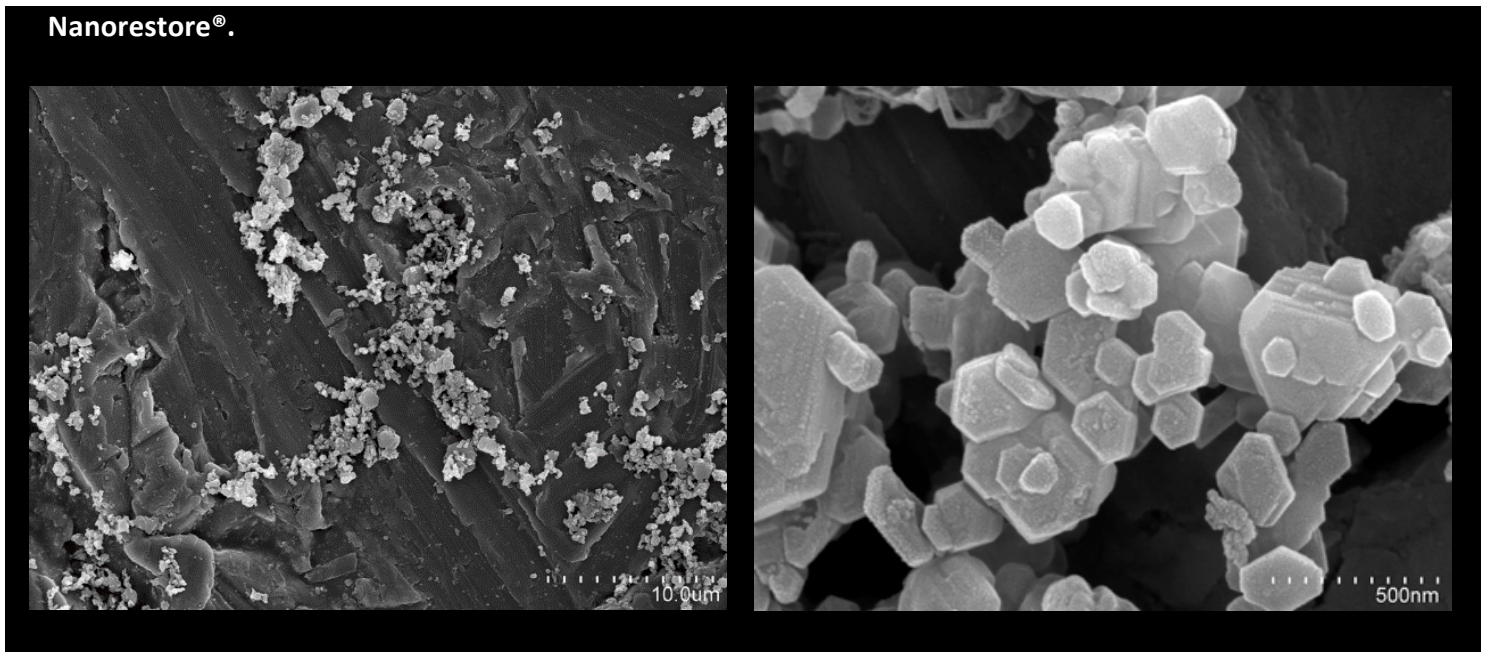

Ilustración 5. Nanorestore ${ }^{\circledR}$. SEM-(Hitachi S4800).

Para estudiar el tratamiento de la adhesión estructural de fragmentos de roca con peligro de desprendimiento, se han elegido: CaLoSiL micro $^{\circledast}$ y CaLoSiL paste like ${ }^{\circledast}$.

El CaLoSiL micro ${ }^{\circledR}$ (Ilustración 6) es una suspensión de $\mathrm{Ca}(\mathrm{OH})_{2}$ en Etanol con una concentración de $120 \mathrm{~g} / \mathrm{l}$, cuyo particulado oscila entre 1 y $3 \mu$. Utilizado para reforzar piedra, morteros y revoques, además de consolidar superficies disgregadas y rellenar grietas y juntas. Admite la adición de áridos en polvo para la preparación de pastas de inyección y morteros de restauración, permitiendo diseñar tratamientos que requieran un control muy específico de la distribución del tamaño de las partículas.

La suspensión coloidal en etanol de CaLoSiL paste-like ${ }^{\circledR}$ (Ilustración 7), por sus características compositivas está recomendado como aglutinante para masas especiales de inyección y morteros de restauración para relleno de pequeñas grietas y huecos.

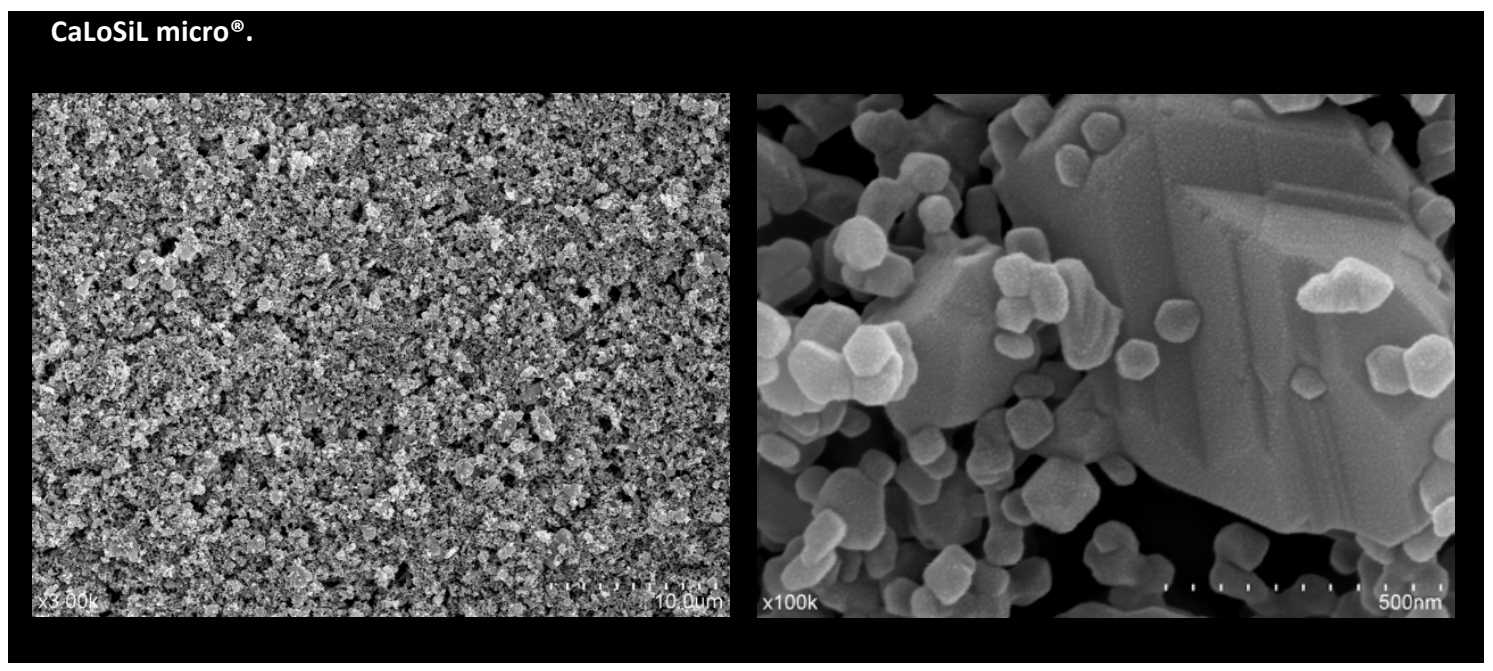

Ilustración 6. CaLoSiL micro ${ }^{\circledR}$. SEM-(Hitachi S4800). 


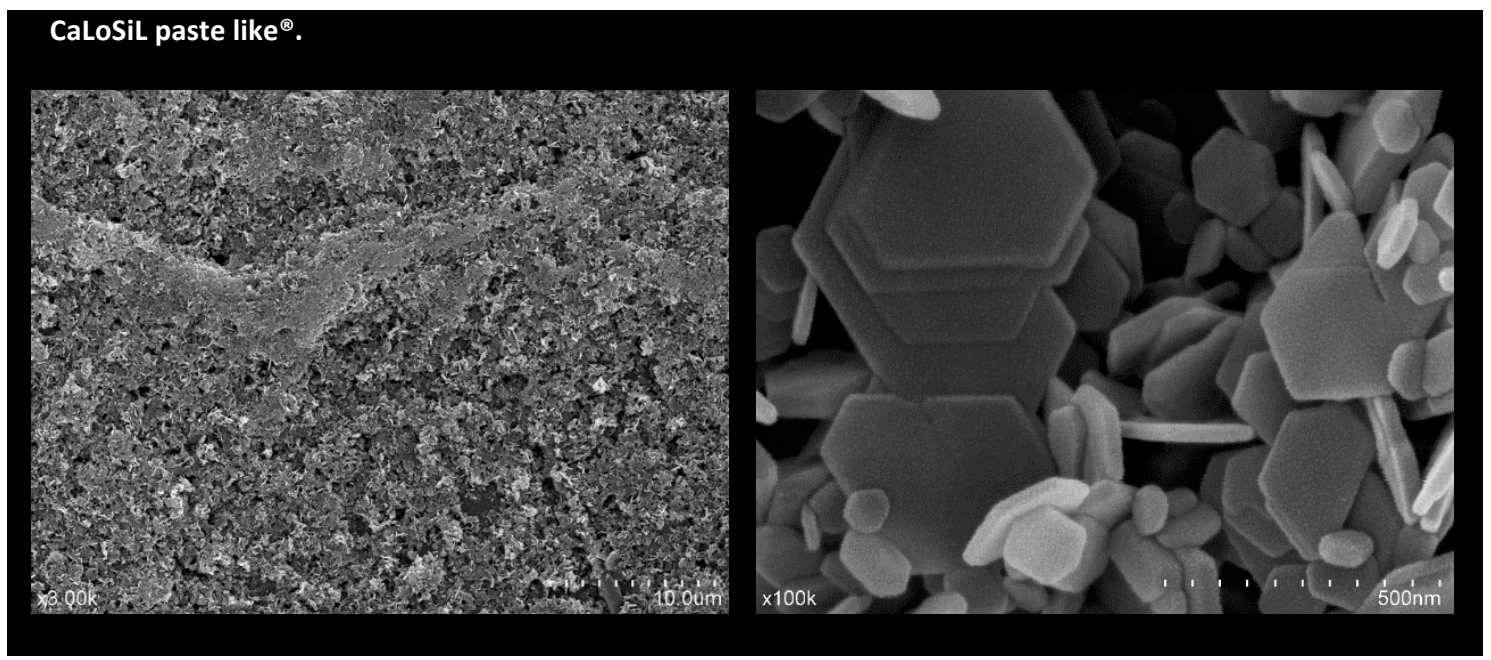

Ilustración 7. CaLoSiL paste like ${ }^{\circledR}$. SEM-(Hitachi S4800).

\section{CONCLUSIONES.}

Con las dispersiones de nanocal en alcohol, se consigue reconstruir la estructura de cristales de carbonato cálcico encargadas de restituir la propiedad de adhesión y cohesión perdida. A diferencia de lo que ocurre con los consolidantes orgánicos como la caseína, o los polímeros sintéticos como el Paraloid $\mathrm{B} 72^{\circledR}$. El estrato pictórico recupera las propiedades mecánicas, pero no mediante la creación de un film polimérico continuo y homogéneo, sino que la recristalización estructural se realiza en forma de puntos de soldadura sin formar films compactos y homogéneos. De modo que, es totalmente compatible químicamente y físicamente con el soporte, del que respeta su porosidad, permeabilidad al $\mathrm{H}_{2} \mathrm{O}$ y transpirabilidad.

Si se establece una comparativa entre los distintos productos a nivel morfológico, topográfico y de distribución espacial de las nanopartículas que lo componen, se identifican claramente tres grupos, según el análisis del particulado visualizado a 100K, mediante microscopia electrónica de barrido SEM-EDX (S-4800):

Un primer grupo formado por CaLoSiL E5 ${ }^{\circledR}, E 25$ grey $^{\circledR}, I P 5^{\circledR}$ y NP5 ${ }^{\circledR}$, en los que se observa que el tamaño y el grosor de los cristales es prácticamente similar; un segundo grupo formado por el Nanorestore ${ }^{\circledR}$ que presenta unas partículas con estructura mucho más fina y laminar, dispuestas espacialmente en agrupaciones puntualizadas, dejando espacios abiertos; y el tercer grupo formado por el CaLoSiL micro y paste like ${ }^{\circledR}$, cuyas partículas son de tamaños de mayor dimensión que los anteriores, su disposición sobre la superficie es muy irregular.

Los resultados obtenidos con análisis (SEM-EDX) determina ciertas variaciones entre los productos: CaLoSiL ${ }^{\circledR}$ y Nanorestore ${ }^{\circledR}$. Éstas pueden deberse al método de síntesis utilizado para la obtención del nanoparticulado. Especialmente la diferencia radica en la forma de las nanopartículas siendo las del Nanorestore ${ }^{\circledR}$ mucho más finas y laminares, con respecto a las que presentan los productos de la gama CaLoSiL ${ }^{\circledR}$. Se observa que en las aplicaciones realizadas con el CaLoSiL IP ${ }^{\circledR}$, el producto crea una superficie homogénea, sin generar acumulaciones o depósitos indeseados. Mientras que el Nanorestore ${ }^{\circledR}$ genera un reticulado regular de micro-concentrados de material disperso sobre la superficie. Probablemente este material requiera de un mayor número de aplicaciones con respecto al CaLoSiL ${ }^{\circledR}$, debido a que sus partículas presentan una morfología diferente de láminas más finas y de menor tamaño. 


\section{FUENTES REFERENCIALES.}

BARREDA, Gemma. "Investigación de tratamientos de consolidación del soporte rocoso en el Abric de Pinos (Benissa-Alicante)", TFM. Universitat Politècnica de València, Valencia. 2012.

BARREDA, Gemma; ZALBIDEA, Ma Antonia. "Consolidants per a suports petris amb manifestacions d'art rupestre a la Comunitat Valenciana. Anàlisi pràctic en Cova Remígia”, UPV, 2016. Disponible en:

https://www.researchgate.net/profile/Mantonia_Munoz/publications?pubType=poster.

BARREDA, Gemma; ZALBIDEA, Mạ Antonia. "Investigación de tratamientos de consolidación del soporte rocoso Abric de Pinos (BenissaAlicante), I Encuentro de Doctorado de la UPV. Valencia, 2014 Disponible en:

https://www.researchgate.net/publication/314975180_INVESTIGACION_DE_TRATAMIENTOS_DE_CONSOLIDACION_DEL_SOPORTE_ROC OSO ABRIC DE PINOS BENISSA-ALICANTE.

BARREDA, Gemma; ZALBIDEA, Ma Antonia. "Estudio comparativo entre consolidantes para soporte pétreo con manifestaciones de arte rupestre mediante ensayos de penetración por tinción” En: EMERGE 2014 - Jornadas de Investigación Emergente en Conservación y Restauración de Patrimonio. UPV. ISBN: 978-84-9048-317-6

DANIELE, Valeria et al. The nanolimes in cultural heritage conservation: characterisation and analysis of the carbonatation process. En Journal of cultural heritage, Vol. 9, no. 3, 2008.

DEI, Luigi et al. Pre-consolidation of pictorial layers in frescoes: The high performance of CSGI's method based on nanolime evaluated by opd team in agnolo gaddi's Leggenda della Vera Croce Paintings, Santa Croce, Florence. En Scienza e Beni Culturali, XXIII. Florence, 2007.

GÓMEZ, Luz Stella et al. Evaluación del tratamiento de consolidación de dolomías mediante nanopartículas de hidróxido de calcio en condiciones de alta humedad relativa. Boletín de la sociedad española de cerámica y vidrio, Vol. 50, no.2, 2011.

GÓMEZ, Luz Stella et al. Aplicación de nanopartículas a la consolidación de patrimonio pétreo. En La ciencia y el arte III: Ciencias experimentales y conservación del patrimonio. 2011.

GÓMEZ, Luz Stella et al. Atomic defects and their relationship to aragonite-calcite transformation in portlandite nanocrystal carbonation. Crystal Growth Design, vol. 12, no. 10, 2012.

GÓMEZ, Luz Stella et al. Carbonatación de Nanocristales de Portlandita obtenidos por síntesis Coloidal: Experiencias sobre su Estabilidad en Rocas Carbonáticas. 2012

GÓMEZ, Luz Stella et al. Nanopartículas para la conservación del patrimonio. La conservación de los geomateriales utilizados en el patrimonio. Madrid: Programa Geomateriales, 2012.

GÓMEZ, Luz Stella et al. La aportación de la nanociencia a la conservación de bienes del patrimonio cultural. Patrimonio cultural de España, no. 4, 2010.

LÓPEZ-ARCE, Paula, et al. Influence of porosity and relative humidity on consolidation of dolostone with calcium hydroxide nanoparticles: effectiveness assessment with non-destructive techniques. Materials Characterization, vol. 61, no. 2, 2010.

OSCA, Julia. Jornada CaLoSiL ${ }^{\circledR}$. Universitat Politècnica de València, Valencia. 2013. 